\title{
Surprises in Correlated Electron Physics
}

\author{
K. Byczuk ${ }^{a, b}$, W. Hofstetter ${ }^{c}$, M. KollaR ${ }^{a}$ \\ AND D. VOLLHARDT ${ }^{a}$ \\ ${ }^{a}$ Theoretical Physics III, Center for Electronic Correlations and Magnetism \\ Institute for Physics, University of Augsburg, 86135 Augsburg, Germany \\ ${ }^{b}$ Institute of Theoretical Physics, Warsaw University \\ Hoża 69, 00-681 Warszawa, Poland \\ ${ }^{c}$ Institut für Theoretische Physik, Johann Wolfgang Goethe-Universität \\ 60438 Frankfurt/Main, Germany \\ Dedicated to Professor Józef Spatek on the occasion of his 60th birthday \\ Strong electronic correlations and especially the interplay between cor- \\ relations and disorder lead to many interesting and quite unexpected phe- \\ nomena. A short summary of our recent investigations into the properties \\ of strongly correlated electron systems with and without disorder using the \\ dynamical mean-field theory is presented.
}

PACS numbers: 71.10.Fd, 71.27.+a, 71.30.+h

\section{Introduction}

The exceptional properties of strongly correlated electron systems have fascinated physicists for several decades already [1-9]. New correlated electron materials and unexpected correlation phenomena are discovered every year. Often the properties of those systems are influenced by disorder. Unfortunately, real materials and even model systems with strong electronic correlations and disorder are notoriously hard to investigate theoretically because standard approximations are invalid in the most interesting parameter regime - that of intermediate coupling. Here the recently developed dynamical mean-field theory (DMFT) [10-17] has proved to be an almost ideal mean-field approximation since it may be used at arbitrary coupling. For this reason the DMFT has been successfully employed in the investigation of electronic correlation effects in theoretical models and even real materials [10-19].

The investigations of electronic correlations and their interplay with disorder by means of the DMFT has led to the discovery of several unexpected properties and phenomena. Examples are: (i) a purely electronic mechanism leading to kinks 
in the electronic dispersion relation [20]; (ii) a novel type of the Mott-Hubbard metal-insulator transition (MIT) away from integer filling in the presence of binary alloy disorder [21]; (iii) an enhancement of the Curie temperature in correlated electron systems with binary alloy disorder [22, 23]; and (iv) unusual effects of correlations and disorder on the Mott-Hubbard and Anderson MITs, respectively $[24,25]$. Below we describe and explain these often surprising results.

The fundamental electronic correlation models investigated here are the Anderson-Hubbard model

$$
H=\sum_{i j, \sigma} t_{i j} c_{i \sigma}^{+} c_{j \sigma}+\sum_{i \sigma} \epsilon_{i} n_{i \sigma}+U \sum_{i} n_{i \uparrow} n_{i \downarrow},
$$

where $t_{i j}$ is the hopping matrix element, $U$ is the local Coulomb interaction, $c_{i \sigma}^{+}$ is the fermionic creation operator for an electron with spin $\sigma$ in the Wannier state $i$, and $n_{i \sigma}$ is the particle number operator; and the Anderson-FalicovKimball model

$$
H=\sum_{i j} t_{i j} c_{i}^{\dagger} c_{j}+\sum_{i} \epsilon_{i} c_{i}^{\dagger} c_{i}+U \sum_{i} f_{i}^{\dagger} f_{i} c_{i}^{\dagger} c_{i},
$$

where $c_{i}^{\dagger}\left(f_{i}^{\dagger}\right)$ and $c_{i}\left(f_{i}\right)$ are fermionic creation and annihilation operators for mobile (immobile) particles at a lattice site $i$. Furthermore, $t_{i j}$ is the hopping amplitude for mobile particles between sites $i$ and $j$, and $U$ is the local interaction energy between mobile and immobile particles occupying the same site. The ionic energy $\epsilon_{i}$ in both models is a random, independent variable which describes the local, quenched disorder affecting the motion of the mobile particles. At large $U$ the models are reduced to $t-J$-like Hamiltonians with spin-exchange interactions between the fermions [26].

The disorder part is modeled by a corresponding probability distribution function (PDF) $P\left(\epsilon_{i}\right)$. For $P\left(\epsilon_{i}\right)=0$ the system is called pure. For binary alloy disorder we assume

$$
P\left(\epsilon_{i}\right)=x \delta\left(\epsilon_{i}+\frac{\Delta}{2}\right)+(1-x) \delta\left(\epsilon_{i}-\frac{\Delta}{2}\right),
$$

where $\Delta$ is the energy difference between the two atomic energies which provides a measure of the disorder strength, while $x$ and $1-x$ are the concentrations of the two alloy atoms. Another model of disorder which we use is one with the continuous PDF

$$
P\left(\epsilon_{i}\right)=\frac{\Theta\left(\frac{\Delta}{2}-\left|\epsilon_{i}\right|\right)}{\Delta}
$$

with $\Theta$ as the step function. Again the parameter $\Delta$ is a measure of the disorder strength.

\section{Kinks in the electronic dispersion}

Dispersion relations characterize quasiparticles and collective excitations of many-body systems. The coupling between them may lead to kinks, i.e. abrupt changes in the slope of the dispersion. Such kinks thus carry important informa- 
tion about interactions in a many-body system. Recently we have found a novel, purely electronic mechanism yielding kinks in the electron dispersions [20]. This mechanism does not require a coupling of two different excitations as in previously known cases. Our theory applies to strongly correlated metals whose spectral function shows well separated Hubbard subbands and central peak as, for example, in transition metal-oxides.

For a microscopic description of these electronic kinks we use the Hubbard model (1) without disorder. For simplicity, we focus here on a single band with a particle-hole symmetry. The model was solved using the DMFT at $T=0$ [20].

The effective dispersion relation $E_{\boldsymbol{k}}$ of the one-particle excitation is determined by the singularities of $G(\boldsymbol{k}, \omega)=\left[\omega+\mu-\epsilon_{\boldsymbol{k}}-\Sigma(\boldsymbol{k}, \omega)\right]^{-1}$, which give rise to peaks in the spectral function $A(\boldsymbol{k}, \omega)=-\operatorname{Im} G(\boldsymbol{k}, \omega) / \pi$. Here $\omega$ is the frequency, $\mu$ - the chemical potential, $\epsilon_{\boldsymbol{k}}$ - the bare dispersion relation, and $\Sigma(\boldsymbol{k}, \omega)$ is the self-energy. If the damping given by the imaginary part of $\Sigma(\boldsymbol{k}, \omega)$ is not too large, the effective dispersion $E_{\boldsymbol{k}}$ is thus determined by

$$
E_{\boldsymbol{k}}+\mu-\epsilon_{\boldsymbol{k}}-\operatorname{Re} \Sigma\left(\boldsymbol{k}, E_{\boldsymbol{k}}\right)=0 .
$$

Any kinks in $E_{\boldsymbol{k}}$ that do not originate from $\epsilon_{\boldsymbol{k}}$ must therefore be due to changes in the slope of $\operatorname{Re} \Sigma(\boldsymbol{k}, \omega)$.

We use the DMFT self-consistency equations to express $\Sigma(\boldsymbol{k}, \omega)=\Sigma(\omega)$ as $\Sigma(\omega)=\omega+\mu-1 / G(\omega)-\Delta(G(\omega))$, where $G(\omega)=\int G(\boldsymbol{k}, \omega) \mathrm{d} \boldsymbol{k}$ is the local Green function (averaged over $\boldsymbol{k}$ ) and $\Delta(G)$ is an energy-dependent hybridization function, expressed here as a function of $G(\omega)$.

Kinks in $\operatorname{Re} \Sigma(\omega)$ appear at a new small energy scale which emerges quite generally for a three-peak spectral function $A(\omega)$, cf. Fig. 1. The KramersKronig relations imply that $\operatorname{Re} G(\omega)$ is small near the dips of $A(\omega)$, located at $\pm \Omega$. Therefore, $\operatorname{Re} G(\omega)$ has a maximum and a minimum at $\pm \omega_{\max }$ inside the central spectral peak (Fig. 1b). This directly leads to kinks in $\operatorname{Re} \Sigma(\omega)$ for the following reason. There are two contributions to $\Sigma(\omega)$ : $\omega+\mu-1 / G(\omega)$ and $-\Delta(G(\omega))$. While $\operatorname{Re}(\omega+\mu-1 / G(\omega))$ is linear in the large energy window $|\omega|<\Omega$ (Fig. 1d), the term $-\operatorname{Re} \Delta(G(\omega))$ is approximately proportional to $\operatorname{Re} G(\omega)$ (at least to the first order in a moment expansion), and thus remains linear only in a much narrower energy window $|\omega|<\omega_{\max }$. The sum of these two contributions produces pronounced kinks in the real part of the self-energy at $\pm \omega_{*}$, where $\omega_{*}=0.41 \omega_{\max }$ is the energy, where $\operatorname{Re} G(\omega)$ has maximum curvature (marked by circles in Fig. 1c). The Fermi-liquid (FL) regime with slope $\partial \operatorname{Re} \Sigma(\omega) / \partial \omega=1-1 / Z_{\mathrm{FL}}$ thus extends only throughout a small part of the central peak $\left(|\omega|<\omega_{*}\right)$. At intermediate energies $\left(\omega_{*}<|\omega|<\Omega\right)$ the slope is then given by $\partial \operatorname{Re} \Sigma(\omega) / \partial \omega=1-1 / Z_{\mathrm{CP}}$, where $\mathrm{CP}$ stands for the central peak. The kinks at $\pm \omega_{*}$ mark the crossover between these two slopes. As a consequence, there is also a kink at $\omega_{*}$ in the effective band structure $E_{\boldsymbol{k}}$.

The FL regime terminates at the kink energy scale $\omega_{*}$, which cannot be determined within FL theory itself. The quantities $\omega_{*}$ and $Z_{\mathrm{CP}}$ can all nevertheless 

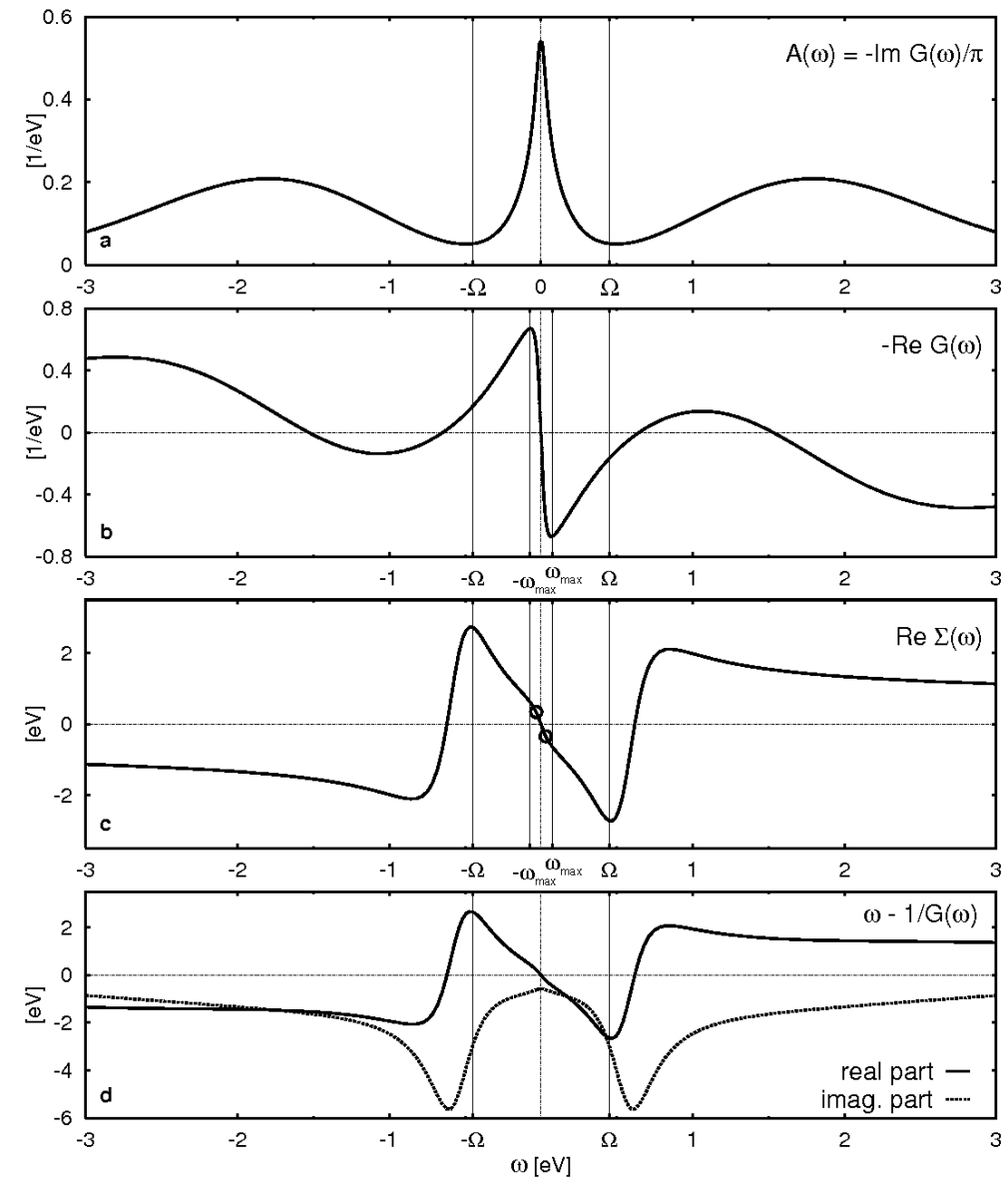

Fig. 1. Local propagator and self-energy for a strongly correlated system. (a) Correlation-induced three-peak spectral function $A(\omega)=-\operatorname{Im} G(\omega) / \pi$ with dips at $\pm \Omega=$ $0.45 \mathrm{eV}$. (b) Corresponding real part of the propagator, $-\operatorname{Re} G(\omega)$, with minimum and maximum at $\pm \omega_{\max }$ inside the central spectral peak. (c) Real part of the selfenergy with kinks at $\pm \omega_{*}$ (circles), located at the points of maximum curvature of $\operatorname{Re} G(\omega)\left(\omega_{*}=0.4 \omega_{\max }=0.03 \mathrm{eV}\right)$. (d) $\omega-1 / G(\omega)$ contributes to the self-energy. In general $\operatorname{Re}(\omega-1 / G(\omega))$ is linear in $|\omega|<\Omega$. The other contribution to the self-energy is $-\Delta(G(\omega)) \approx-\left(m_{2}-m_{1}^{2}\right) G(\omega)$ (to the lowest order in the moments $m_{i}$ of $\epsilon_{\boldsymbol{k}}$; here $\left.m_{2}-m_{1}^{2}=0.5 \mathrm{eV}^{2}\right)$. Therefore, the nonlinearity of $-\operatorname{Re} G(\omega)$ at $\pm \omega_{*}$ determines the location of kinks. (Hubbard model in DMFT, cubic lattice, interaction $U=3.5 \mathrm{eV}$, band width $W \approx 3.46 \mathrm{eV}, n=1, Z_{\mathrm{FL}}=0.086$; after Ref. [20]).

be expressed in terms of $Z_{\mathrm{FL}}$ and the bare density of states alone; explicitly, one finds $\omega_{*}=0.41 Z_{\mathrm{FL}} D$, where $D$ is an energy scale of the noninteracting system, e.g., $D$ is approximately given by half the band width [20]. 
The energy scale $\omega_{*}$ involves only the bare band structure which can be obtained, for example, from band structure calculations, and the FL renormalization $Z_{\mathrm{FL}}=1 /(1-\partial \operatorname{Re} \Sigma(0) / \partial \omega) \equiv m / m^{*}$ known from, e.g., specific heat measurements or many-body calculations. We note that since phonons are not involved in this mechanism, $\omega_{*}$ shows no isotope effect. For strongly interacting systems, in particular close to a metal-insulator transition, $\omega_{*}$ can become quite small, e.g., smaller than the Debye energy.

\section{Metal-insulator transition at fractional filling}

The Mott-Hubbard MIT occurs upon increasing the interaction strength $U$ in the models (1) and (2) if the number of electrons $N_{\mathrm{e}}$ is commensurate with the number of lattice sites $N_{\mathrm{L}}$ or, more precisely, if the ratio $N_{\mathrm{e}} / N_{\mathrm{L}}$ is an odd integer. At zero temperature it is a continuous transition whereas at finite temperatures the transition is of the first order [27, 17]. Surprisingly, in the presence of binary alloy disorder the MIT occurs at fractional filling [21].

We describe this situation by using the Anderson-Hubbard model (1) with the distribution (3) which corresponds to a binary-alloy system composed of two different atoms $\mathrm{A}$ and $\mathrm{B}$. The atoms are distributed randomly on the lattice and have ionic energies $\epsilon_{\mathrm{A}, \mathrm{B}}$, with $\epsilon_{\mathrm{B}}-\epsilon_{\mathrm{A}}=\Delta$. The concentration of $\mathrm{A}(\mathrm{B})$ atoms is given by $x=N_{\mathrm{A}} / N_{\mathrm{L}}\left(1-x=N_{\mathrm{B}} / N_{\mathrm{L}}\right)$, where $N_{\mathrm{A}}\left(N_{\mathrm{B}}\right)$ is the number of the corresponding atoms.

From the localization theorem (the Hadamard-Gerschgorin theorem in matrix algebra) it is known that if the Hamiltonian (1), with a binary alloy distribution for $\epsilon_{i}$, is bounded, then there is a gap in the single-particle spectrum for sufficiently large $\Delta \gg \max (|t|, U)$. Hence at $\Delta=\Delta_{\mathrm{c}}$ the density of states (DOS) splits into two parts corresponding to the lower and the upper alloy subbands with centers of mass at the ionic energies $\epsilon_{\mathrm{A}}$ and $\epsilon_{\mathrm{B}}$, respectively. The width of the alloy gap is of the order of $\Delta$. The lower and upper alloy subband contains $2 x N_{\mathrm{L}}$ and $2(1-x) N_{\mathrm{L}}$ states, respectively.

New possibilities appear in systems with correlated electrons and binary alloy disorder [21]. The Mott-Hubbard metal-insulator transition can occur at any filling $n=x$ or $1+x$, corresponding to a half-filled lower or to a half-filled upper alloy subband, respectively, as shown schematically for $n=x$ in Fig. 2. The Mott insulator can then be approached either by increasing $U$ when $\Delta \geq \Delta_{\mathrm{c}}$ (alloy band splitting limit), or by increasing $\Delta$ when $U \geq U_{\mathrm{c}}$ (Hubbard band splitting limit). The nature of the Mott insulator in the binary alloy system can be understood physically as follows. Due to the high energy cost of the order of $U$ the randomly distributed ions with lower (higher) local energies $\epsilon_{i}$ are singly occupied at $n=x(n=1+x)$, i.e., the double occupancy is suppressed. In the Mott insulator with $n=x$ the ions with higher local energies are empty and do not contribute to the low-energy processes in the system. Likewise, in the Mott 


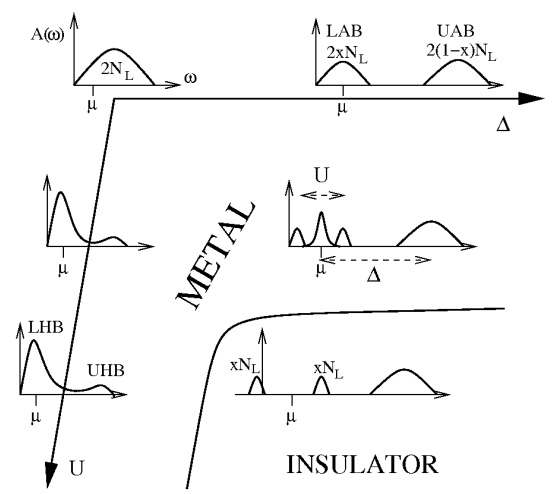

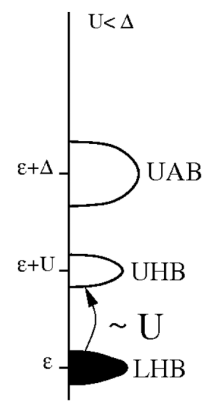

alloy Mott insulator

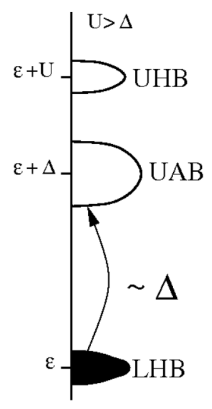

alloy charge transfer insulator

Fig. 2. Left: Schematic plot representing the Mott-Hubbard metal-insulator transition in a correlated electron system with the binary alloy disorder. The shapes of spectral functions $A(\omega)$ are shown for different interactions $U$ and disorder strengths $\Delta$. Increasing $\Delta$ at $U=0$ leads to splitting of the spectral function into the lower (LAB) and the upper (UAB) alloy subbands, which contain $2 x N_{\mathrm{L}}$ and $2(1-x) N_{\mathrm{L}}$ states, respectively. Increasing $U$ at $\Delta=0$ leads to the occurrence of lower (LHB) and upper (UHB) Hubbard subbands. The Fermi energy for filling $n=x$ is indicated by $\mu$. At $n=x$ (or $n=1+x$, not shown in the plot) the LAB (UAB) is half-filled. In this case an increase in $U$ and $\Delta$ leads to the opening of a correlation gap at the Fermi level and the system becomes a Mott insulator. Right: Two possible insulating states in the correlated electron system with binary-alloy disorder. When $U<\Delta$ the insulating state is an alloy Mott insulator with an excitation gap in the spectrum of the order of $U$. When $U>\Delta$ the insulating state is an alloy charge transfer insulator with an excitation gap of the order of $\Delta$; after Ref. [21].

insulator with $n=1+x$ the ions with lower local energies are double occupied implying that the lower alloy subband is blocked and does not play any role.

For $U>U_{\mathrm{c}}(\Delta)$ in the Mott insulating state with binary alloy disorder one may use the lowest excitation energies to distinguish two different types of insulators. Namely, for $U<\Delta$ an excitation must overcome the energy gap between the lower and the upper Hubbard subbands, as indicated in Fig. 2. We call this insulating state an alloy Mott insulator. On the other hand, for $\Delta<U$ an excitation must overcome the energy gap between the lower Hubbard subband and the upper alloy-subband, as shown in Fig. 2. We call this insulating state an alloy charge transfer insulator.

In Fig. 3 we present a particular phase diagram for the Anderson-Hubbard model at filling $n=0.5$ showing a Mott-Hubbard type of MIT with a typical hysteresis. 


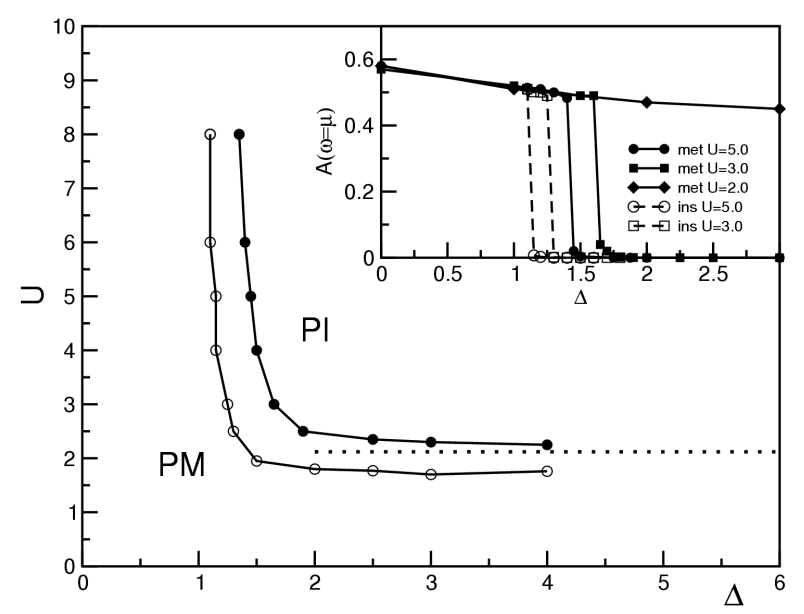

Fig. 3. Ground state phase diagram of the Hubbard model with binary-alloy disorder at filling $n=x=0.5$. The filled (open) dots represent the boundary between paramagnetic metallic (PM) and paramagnetic insulating (PI) phases as determined by DMFT with the initial input given by the metallic (insulating) hybridization function. The horizontal dotted line represents $U_{\mathrm{c}}$ obtained analytically from an asymptotic theory in the limit $\Delta \rightarrow \infty$. Inset: hysteresis in the spectral functions at the Fermi level obtained from DMFT with an initial metallic (insulating) host represented by filled (open) symbols and solid (dashed) lines; after Ref. [21].

\section{Disorder-induced enhancement of the Curie temperature}

Itinerant ferromagnetism in the pure Hubbard model occurs only away from half-filling and if the DOS is asymmetric and peaked at the lower edge [28, 29]. While the Curie temperature increases with the strength of the electron interaction one would expect it to be lowered by disorder. However, our investigations show that in some cases the Curie temperature can actually be increased by binary alloy disorder $[22,23]$.

Indeed, the Curie temperature as a function of alloy concentration exhibits a very rich and interesting behavior as is shown in Fig. 4. At some concentrations and certain values of $U, \Delta$, and $n$, the Curie temperature is enhanced above the corresponding value for the non-disordered case $(x=0$ or 1$)$. This is shown in the upper part of Fig. 4 for $0<x<0.2$. The relative increase in $T_{\mathrm{c}}$ can be as large as $25 \%$, as is found for $x \approx 0.1$ at $n=0.7, U=2$ and $\Delta=4$ (upper part of Fig. 4).

This unusual enhancement of $T_{\mathrm{c}}$ is caused by three distinct features of interacting electrons in the presence of binary alloy disorder:

i) The Curie temperature in the non-disordered case $T_{\mathrm{c}}^{\mathrm{p}} \equiv T_{\mathrm{c}}(\Delta=0)$, depends non-monotonically on band filling $n$ [28]. Namely, $T_{\mathrm{c}}^{\mathrm{p}}(n)$ has a maximum at some filling $n=n^{*}(U)$, which increases as $U$ is increased; see also our schematic plots in Fig. 5 . 

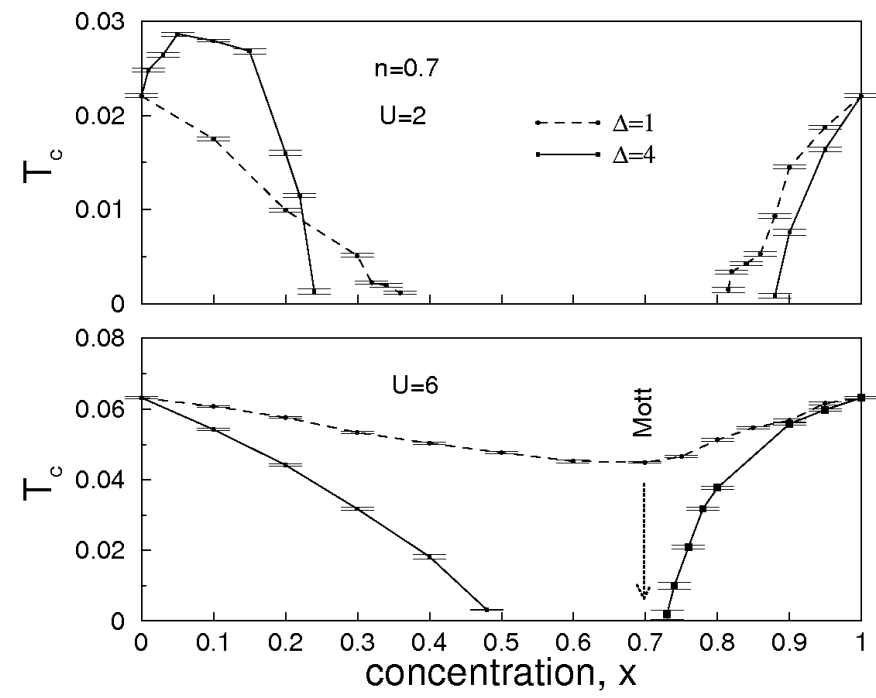

Fig. 4. Curie temperature as a function of alloy concentration $x$ at $U=2$ (upper part) and 6 (lower part) for $n=0.7$ and disorder $\Delta=1$ (dashed lines) and 4 (solid lines); after Refs. [22, 23]. Temperatures are in the energy units.

ii) As was described above, in the alloy-disordered system the band is split when $\Delta \gg W$. As a consequence, for $n<2 x$ and $T \ll \Delta$ electrons occupy only the lower alloy subband and for $n>2 x$ both the lower and upper alloy subbands are filled. In the former case the upper subband is empty while in the latter case the lower subband is completely full. Effectively, one can therefore describe this system by a Hubbard model mapped onto either the lower or the upper alloy subband, respectively. The second subband plays a passive role. Hence, the situation corresponds to a single band with the effective filling $n_{\mathrm{eff}}=n / x$ for $n<2 x$ and $n_{\mathrm{eff}}=(n-2 x) /(1-x)$ for $n>2 x$. It is then possible to determine $T_{\mathrm{c}}$ from the phase diagram of the Hubbard model without disorder.

iii) The disorder leads to a reduction of $T_{\mathrm{c}}^{\mathrm{p}}\left(n_{\mathrm{eff}}\right)$ by a factor $\alpha=x$ if the Fermi level is in the lower alloy subband or $\alpha=1-x$ if it is in the upper alloy subband, i.e. we find

$$
T_{\mathrm{c}}(n) \approx \alpha T_{\mathrm{c}}^{\mathrm{p}}\left(n_{\mathrm{eff}}\right),
$$

when $\Delta \gg W$. Hence, as illustrated in Fig. $5, T_{\mathrm{c}}$ can be determined by $T_{\mathrm{c}}^{\mathrm{p}}\left(n_{\mathrm{eff}}\right)$. Surprisingly, then, it follows that for suitable $U$ and $n$ the Curie temperature of a disordered system can be higher than that of the corresponding non-disordered system (cf. Fig. 5). 

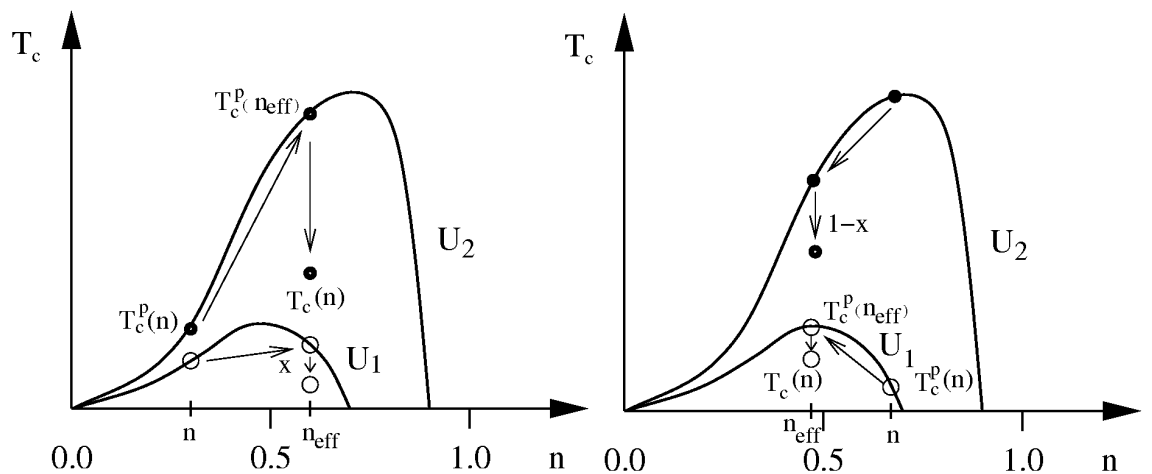

Fig. 5. Schematic plots explaining the filling dependence of $T_{\mathrm{c}}$ for interacting electrons with strong binary alloy disorder. Curves represent $T_{\mathrm{c}}^{\mathrm{p}}$, the Curie temperature for the pure system, as a function of filling $n$ at two different interactions $U_{1} \ll U_{2}$. Left: For $n<x, T_{\mathrm{c}}$ of the disordered system can be obtained by transforming the open (for $U_{1}$ ) and the filled (for $U_{2}$ ) point from $n$ to $n_{\mathrm{eff}}=n / x$, and then multiplying $T_{\mathrm{c}}^{\mathrm{p}}(n / x)$ by $x$ as indicated by arrows. One finds $T_{\mathrm{c}}(n)<T_{\mathrm{c}}^{\mathrm{p}}(n)$ for $U_{1}$, but $T_{\mathrm{c}}(n)>T_{\mathrm{c}}^{\mathrm{p}}(n)$ for $U_{2}$. Right: For $n>x, T_{\mathrm{c}}$ of the disordered system can be obtained by transforming $T_{\mathrm{c}}^{\mathrm{p}}(n)$ from $n$ to $n_{\text {eff }}=(n-2 x) /(1-x)$, and then multiplying $T_{\mathrm{c}}^{\mathrm{p}}[(n-2 x) /(1-x) x]$ by $1-x$ as indicated by arrows. One finds $T_{\mathrm{c}}(n)>T_{\mathrm{c}}^{\mathrm{p}}(n)$ for $U_{1}$, but $T_{\mathrm{c}}(n)<T_{\mathrm{c}}^{\mathrm{p}}(n)$ for $U_{2}$; after Refs. [22, 23].

\section{Continuously connected insulating phases in strongly correlated systems with disorder}

The Mott-Hubbard MIT is caused by the Coulomb correlations in the pure system. By contrast, the Anderson MIT, also referred to as the Anderson localization, is due to coherent backscattering from randomly distributed impurities in a system without interaction [30]. It is therefore a challenge to investigate the effect of the simultaneously presence of interactions and disorder on electronic systems $[24,25]$. In particular, the question arises whether it will suppress or enlarge a metallic phase. And what about the Mott and Anderson insulating phases: will they be separated by a metallic phase? Possible scenarios are schematically plotted in Fig. 6.

The Mott-Hubbard MIT is characterized by the opening of a gap in the density of states at the Fermi level. At the Anderson localization transition the character of the spectrum at the Fermi level changes from a continuous spectrum to a dense, pure point spectrum. It is plausible to assume that both MITs can be characterized by a single quantity, namely, the local density of states (LDOS). Although the LDOS is not an order parameter associated with a symmetry breaking phase transition, it discriminates between a metal and an insulator, which is driven by correlations and disorder, cf. insets to Fig. 6. 


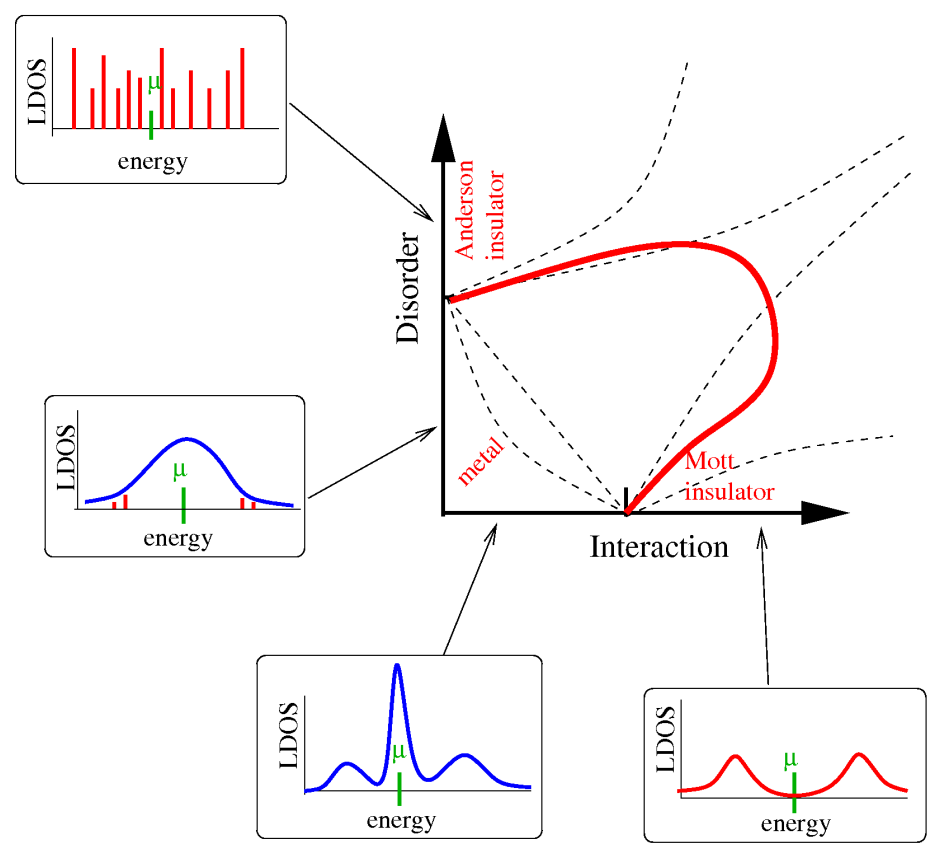

Fig. 6. Possible phases and phase transitions triggered by interaction and disorder in the same system. According to DMFT investigations the simultaneous presence of correlations and disorder enhances the metallic regime (thick line); the two insulating phases are connected continuously. Insets show different local density of states when disorder or interaction is switched off.

In a disordered system the LDOS depends on a particular realization of the disorder in the system. To obtain a full understanding of the effects of disorder it would therefore in principle be necessary to determine the entire probability distribution function of the LDOS, which is almost never possible. Instead one might try to calculate moments of the LDOS. This, however, is insufficient because the arithmetically averaged LDOS (first moment) stays finite at the Anderson MIT [31]. It was already pointed out by Anderson [30] that the "typical" values of random quantities, which are mathematically given by the most probable values of the probability distribution functions, should be used to describe localization. The geometric mean is defined by

$$
A_{\text {geom }}=\exp \left(\left\langle\ln A\left(\epsilon_{i}\right)\right\rangle_{\text {dis }}\right),
$$

and differs from the arithmetical mean given by

$$
A_{\text {arith }}=\left\langle A\left(\epsilon_{i}\right)\right\rangle_{\mathrm{dis}},
$$

where $\left\langle F\left(\epsilon_{i}\right)\right\rangle_{\mathrm{dis}}=\int \mathrm{d} \epsilon_{i} \mathcal{P}\left(\epsilon_{i}\right) F\left(\epsilon_{i}\right)$ is an arithmetic mean of function $F\left(\epsilon_{i}\right)$. The geometrical mean gives an approximation of the most probable ("typical") value of the LDOS and vanishes at a critical strength of the disorder, hence providing an explicit criterion for the Anderson localization [30, 32-34]. 

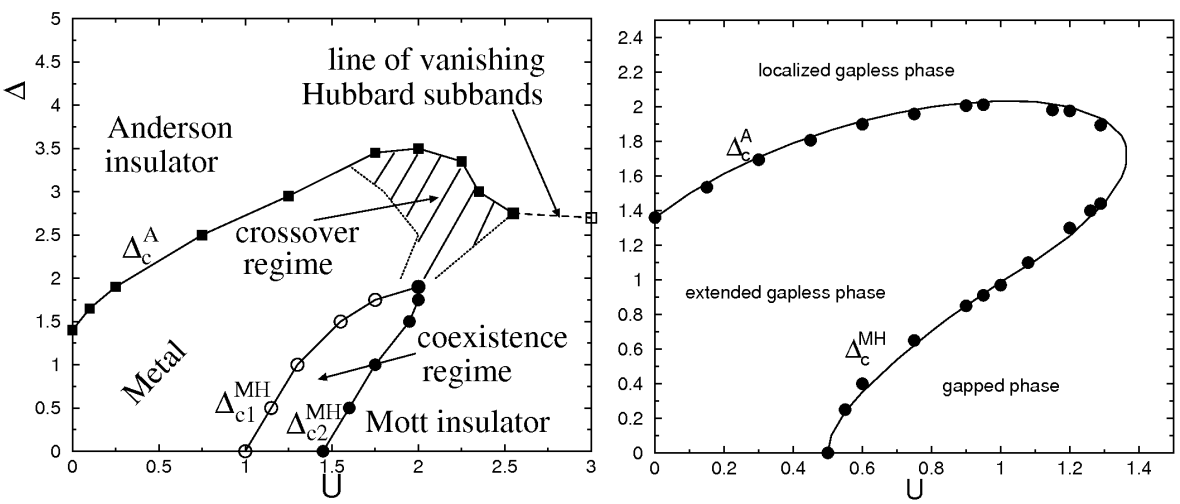

Fig. 7. Non-magnetic ground state phase diagram of the Anderson-Hubbard (left) and Anderson-Falicov-Kimball (right) models at half-filling as calculated by DMFT with the typical local density of states; after Refs. [24, 25].

A non-perturbative framework for investigations of the Mott-Hubbard MIT in lattice electrons with a local interaction and disorder is provided by the DMFT $[17,15]$. If in this approach the effect of local disorder is taken into account through the arithmetic mean of the LDOS [35] one obtains, in the absence of interactions, the well-known coherent potential approximation (CPA) [36], which does not describe the physics of Anderson localization. To overcome this deficiency Dobrosavljević et al. [33] incorporated the geometrically averaged LDOS into the self-consistency cycle and thereby derived a mean-field theory of Anderson localization which reproduces many of the expected features of the disorder-driven MIT for non-interacting electrons. This scheme uses only one-particle quantities and is therefore easily incorporated into the DMFT for disordered electrons in the presence of phonons [37], or Coulomb correlations [24, 25]. In particular, the DMFT with geometrical averaging allows to compute phase diagrams for the Anderson-Hubbard model (1) and the Anderson-Falicov-Kimball model (2) with the continuous probability distribution function (4) at half-filling [24, 25]. In this way we found that, although in both models the metallic phase is enhanced for small and intermediate values of the interaction and disorder, metallicity is finally destroyed. Surprisingly, the Mott and Anderson insulators are found to be continuously connected. Phase diagrams for the non-magnetic ground state are shown in Fig. 7.

\section{Conclusions}

The physics of correlated electron systems is known to be extremely rich. Therefore their investigation continues to unravel novel and often surprising phenomena. The presence of disorder further enhances this complexity. Here we discussed several remarkable features induced by correlations with and without 
disorder, which came as a surprise when they were first discovered, but which after all have physically intuitive explanations.

\section{Acknowledgments}

It is a pleasure to thank R. Bulla, K. Held, I.A. Nekrasov, Th. Pruschke, M. Ulmke, and Y.-F. Yang for discussions and collaborations. This work was supported by the Sonderforschungsbereich 484 of the Deutsche Forschungsgemeinschaft.

\section{References}

[1] J.H. de Boer, E.J.W. Verwey, Proc. Phys. Soc. 49, No. 4S, 59 (1937).

[2] N.F. Mott, Proc. Phys. Soc. 49, No. 4S, 57 (1937).

[3] D. Pines, The Many-Body Problem, W.A. Benjamin, Reading 1962.

[4] N.F. Mott, Metal-Insulator Transitions, Taylor and Francis, London 1990.

[5] P. Fulde, Electron Correlations in Molecules and Solids, Springer, Heidelberg 1995.

[6] F. Gebhard, The Mott Metal-Insulator Transition, Springer, Heidelberg 1997.

[7] M. Imada, A. Fujimori, Y. Tokura, Rev. Mod. Phys. 70, 1039 (1998).

[8] P. Fazekas, Lecture Notes on Electron Correlation and Magnetism, World Scientific, Singapore 1999.

[9] J. Spalek, Eur. J. Phys. 21, 511 (2000).

[10] W. Metzner, D. Vollhardt, Phys. Rev. Lett. 62, 324 (1989).

[11] E. Müller-Hartmann, Z. Phys. B 76, 211 (1989).

[12] V. Janiš, Z. Phys. B 83, 227 (1991); V. Janiš, D. Vollhardt, Int. J. Mod. Phys. 6, 731 (1992).

[13] A. Georges, G. Kotliar, Phys. Rev. B 45, 6479 (1992).

[14] M. Jarrell, Phys. Rev. Lett. 69, 168 (1992).

[15] D. Vollhardt, in: Correlated Electron Systems, Ed. V.J. Emery, World Scientific, Singapore 1993, p. 57.

[16] Th. Pruschke, M. Jarrell, J.K. Freericks, Adv. Phys. 44, 187 (1995).

[17] A. Georges, G. Kotliar, W. Krauth, M.J. Rozenberg, Rev. Mod. Phys. 68, 13 (1996).

[18] G. Kotliar, D. Vollhardt, Physics Today 57, 53 (2004).

[19] G. Kotliar, S.Y. Savrasov, K. Haule, V.S. Oudovenko, O. Parcollet, C.A. Marianetti, Rev. Mod. Phys. 78, 865 (2006).

[20] K. Byczuk, M. Kollar, K. Held, Y.-F. Yang, I.A. Nekrasov, Th. Pruschke, D. Vollhardt, Nature Phys. 3, 168 (2007), doi:10.1038/nphys538 (2007) [cond-mat/0609594].

[21] K. Byczuk, W. Hofstetter, D. Vollhardt, Phys. Rev. B 69, 045112 (2004).

[22] K. Byczuk, M. Ulmke, D. Vollhardt, Phys. Rev. Lett. 90, 196403 (2003).

[23] K. Byczuk, M. Ulmke, Eur. Phys. J. B 45, 449 (2005).

[24] K. Byczuk, W. Hofstetter, D. Vollhardt, Phys. Rev. Lett. 94, 056404 (2005). 
[25] K. Byczuk, Phys. Rev. B 71, 205105 (2005).

[26] K.A. Chao, J. Spalek, A.M. Oles, J. Phys. C 10, L271 (1977).

[27] J. Spalek, A. Datta, J.M. Honig, Phys. Rev. Lett. 59, 728 (1987).

[28] M. Ulmke, Eur. Phys. J. B 1, 301 (1998).

[29] J. Wahle, N. Blumer, J. Schlipf, K. Held, D. Vollhardt, Phys. Rev. B 58, 12749 (1998).

[30] P.W. Anderson, Phys. Rev. 109, 1492 (1958).

[31] D. Lloyd, J. Phys. C 2, 1717 (1969); D. Thouless, Phys. Rep. 13, 93 (1974); F. Wegner, Z. Phys. B 44, 9 (1981).

[32] V. Dobrosavljević, G. Kotliar, Phys. Rev. Lett. 78, 3943 (1997).

[33] V. Dobrosavljević, A.A. Pastor, B.K. Nikolić, Europhys. Lett. 62, 76 (2003).

[34] G. Schubert, A. Weiße, H. Fehske, cond-mat/0309015.

[35] V. Janiš, D. Vollhardt, Phys. Rev. B 46, 15712 (1992); M. Ulmke, V. Janiš, D. Vollhardt, Phys. Rev. B 51, 10411 (1995).

[36] R. Vlaming, D. Vollhardt, Phys. Rev. B 45, 4637 (1992).

[37] F.X. Bronold, A. Alvermann, H. Fehske, Philos. Mag. 84, 637 (2004). 